\title{
THERMAL STABILITY OF GaN INVESTIGATED BY RAMAN SCATTERING
}

\author{
M. Kuball ${ }^{(1)}$, F. Demangeot ${ }^{(2)}$, J. Frandon ${ }^{(2)}$, M.A. Renucci ${ }^{(2)}$, N. Grandjean ${ }^{(3)}$, and O. Briot ${ }^{(4)}$ \\ ${ }^{(1)}$ H.H. Wills Physics Laboratory, University of Bristol, Bristol BS8 1TL, UNITED KINGDOM \\ ${ }^{(2)}$ Laboratoire de Physique des Solides de Toulouse, CNRS, Université Paul Sabatier, \\ F-31062 Toulouse Cedex, FRANCE \\ ${ }^{(3)}$ CRHEA-CNRS, Rue Bernard Gregory, F-06560 Valbonne, FRANCE \\ ${ }^{(4)}$ GES-CNRS, CC074 Université Montpellier II, Place E.Bataillon \\ F-34095 Montpellier Cedex 5, FRANCE
}

Cite this article as: MRS Internet J. Nitride Semicond. Res. 4S1, G6.28

\begin{abstract}
We have investigated the thermal stability of GaN using Raman scattering. Noninvasive optical monitoring of the degradation of $\mathrm{GaN}$ during high-temperature processing has been demonstrated. GaN samples grown by molecular-beam epitaxy (MBE) and metalorganic vapor phase epitaxy (MOCVD) were studied. Characteristic features in the Raman spectrum identify three thermal stability regimes: (1) annealing below $900^{\circ} \mathrm{C}$ does not affect the GaN Raman spectrum; (2) annealing between $900^{\circ} \mathrm{C}$ and $1000^{\circ} \mathrm{C}$ results in the appearance of disorderinduced Raman scattering between the $\mathrm{E}_{2}$ and $\mathrm{A}_{1}(\mathrm{LO})$ phonon; (3) annealing at temperatures higher than $1000^{\circ} \mathrm{C}$ gives rise to distinct Raman modes at $630 \mathrm{~cm}^{-1}, 656 \mathrm{~cm}^{-1}$ and $770 \mathrm{~cm}^{-1}$. The evolution of the Raman spectrum of $\mathrm{GaN}$ with increasing annealing temperature is discussed in terms of disorder-induced Raman scattering. We find clear indications for an interfacial reaction between $\mathrm{GaN}$ and sapphire for annealing temperatures higher than $1000^{\circ} \mathrm{C}$.
\end{abstract}

\section{INTRODUCTION}

The family of III-V nitrides ( $\mathrm{GaN}$, InGaN, AlGaN) has recently attracted much attention because of their wide spectrum of potential applications ranging from opto-electronic devices for the blue-ultraviolet spectral region to high temperature devices [1,2]. High-temperature processing of nitrides is an essential part of the nitride device fabrication, i.e., for the activation of Mg-acceptors to achieve p-doping of $\mathrm{GaN}$ or for the fabrication of ohmic contacts $[3,4]$. Monitoring the thermal stability of GaN during high-temperature annealing is therefore of great interest [5-7]. Since changes in the near-surface stoichiometry that deteriorate electrical properties occur at a temperature, which is about $100^{\circ} \mathrm{C}$ lower than the temperature where visible surface degradation occurs, it is essential to develop effective non-invasive ex-situ as well as insitu monitoring techniques to assess changes in the quality of $\mathrm{GaN}$ and $\mathrm{AlGaN}$ layers at elevated temperatures. So far only time-consuming techniques like atomic force microscopy (AFM) and transmission electron microscopy (TEM) have been used to assess degradation effects [6,7].

In this report, we illustrate the potential applications of Raman scattering (complemented by photoluminescence experiments) to determine and monitor the degradation pathway of GaN during high-temperature annealing. The results of this study can be employed for the noninvasive optical monitoring of structural damage to GaN during high-temperature processing as well as for the non-invasive optical monitoring of passive and active nitride devices operating at elevated temperatures. Raman scattering provides information on vibrational states of GaN, 
which are sensitive to microscopic disorder. The crystalline quality can be judged from the peak shapes and the selection rules. GaN normally crystallize in the hexagonal wurtzite structure (space group $\mathrm{C}_{6 \mathrm{v}}{ }^{4}$ ) with four atoms in the unit cell. At $\mathbf{k}=\mathbf{0}$ group theory predicts the following eight sets of phonon modes: $2 \mathrm{~A}_{1}+2 \mathrm{~B}_{1}+2 \mathrm{E}_{1}+2 \mathrm{E}_{2}$ of which only one $\mathrm{A}_{1}$, one $\mathrm{E}_{1}$ and two $\mathrm{E}_{2}$ are Raman active. One set of $A_{1}$ and one set of $E_{1}$ corresponds to acoustic phonons, the $B_{1}$ modes are silent [8]. The activation of $\mathbf{q} \neq \mathbf{0}$ phonons of $\mathrm{GaN}$ by disorder allows us to probe and assess noninvasively changes in the crystalline quality of GaN. We find clear indications for a interfacial reaction between sapphire and $\mathrm{GaN}$ at elevated temperatures.

\section{EXPERIMENT}

Raman scattering and photoluminescence spectra were recorded from high-temperature annealed $\mathrm{GaN}$ layers. The samples were annealed in nitrogen ambient in a heating stage at temperatures from $100^{\circ} \mathrm{C}$ to $1100^{\circ} \mathrm{C}$ in $50-100^{\circ} \mathrm{C}$ steps, in a sequence of $20 \mathrm{~min}$ anneals. A small amount of oxygen was present during the annealing. Raman spectra were collected after each annealing step on a Renishaw Raman microscope system 2000 using the $488 \mathrm{~nm}$ line of an $\mathrm{Ar}^{+}-$ laser as excitation source. Photoluminescence spectra were recorded using a Renishaw ultraviolet (UV) Raman microscope system with the 244nm line of an intra-cavity frequency doubled $\mathrm{Ar}^{+}$ laser as excitation source. The Raman measurements were performed in backscattering $\mathrm{Z}(\mathrm{Y}, \mathrm{Y}) \square$ geometry, however, when necessary a full polarization analysis was performed. The studied GaN samples, $2 \mu \mathrm{m}$ thick, were grown by molecular-beam epitaxy (MBE) and metalorganic vapor phase epitaxy (MOCVD) at growth temperatures of $980-1150^{\circ} \mathrm{C}$ on sapphire [0001] substrates, after a thin buffer layer of low temperature $\mathrm{GaN}$ had been deposited at $550-600^{\circ} \mathrm{C}$. The $\mathrm{MBE}$ sample was doped with $\mathrm{Mg}$, annealed in nitrogen ambient at $750^{\circ} \mathrm{C}$ for $30 \mathrm{~min}$, with a free carrier concentration of about $3 \times 10^{17} \mathrm{~cm}^{-3}$ and a mobility of $8 \mathrm{~cm}^{2} / \mathrm{Vs}$. The MOCVD sample was unintentionally n-doped.

\section{RESULTS}

\section{$\underline{\text { Raman scattering }}$}

Figure 1 shows Raman spectra of the MBE-grown GaN layer after various 20min anneals, recorded at room temperature in backscattering $Z Y, Y) \underline{Z}$ geometry. The Raman spectrum shows before annealing (lowest spectrum) the allowed $E_{2}$ (high) phonon at $567 \mathrm{~cm}^{-1}$ and the $\mathrm{A}_{1}(\mathrm{LO})$ phonon at $734 \mathrm{~cm}^{-1}$. We observe at $534 \mathrm{~cm}^{-1}$ the $\mathrm{A}_{1}(\mathrm{TO})$ mode which is usually not allowed in this configuration, however, might be related to a high defect density or the p-doping. The mode at $750 \mathrm{~cm}^{-1}$ is a reminiscent of the $E_{\mathrm{g}}$ sapphire mode. The shown spectra are normalized to the $\mathrm{E}_{2}$ phonon intensity.

The Raman spectrum of the MBE-grown GaN layer remains unchanged for annealing temperatures up to $800-900^{\circ} \mathrm{C}$. Heat treatments at temperatures between $900^{\circ} \mathrm{C}$ to $1000^{\circ} \mathrm{C}$, however, result in a broad Raman peak, centered at $610 \mathrm{~cm}^{-1}$, between the $E_{2}$ and $A_{1}(L O)$ phonon lines. A polarization analysis reveals that this new feature in the Raman spectrum is present in parallel and in crossed polarizations $[Z(Y, Y) \underline{Z}, Z(X, Y) \underline{Z}]$. The $E_{2}$ phonon shifts from $567 \mathrm{~cm}^{-1}$ before annealing to $570 \mathrm{~cm}^{-1}$ after annealing at $1000^{\circ} \mathrm{C}$ (inset of Fig. 1), whereas the $\mathrm{A}_{1}(\mathrm{LO})$ phonons remains unaffected within the experimental spectral resolution.

Annealing the sample at a temperature higher than $1000^{\circ} \mathrm{C}$ results in distinct Raman peaks at $630 \mathrm{~cm}^{-1}, 656 \mathrm{~cm}^{-1}$ and $770 \mathrm{~cm}^{-1}$. Their intensity increases in the subsequent annealing steps. The $\mathrm{A}_{1}(\mathrm{LO})$ phonon at $734 \mathrm{~cm}^{-1}$ broadens and decreases in intensity. The $\mathrm{E}_{1}(\mathrm{TO})$ phonon 


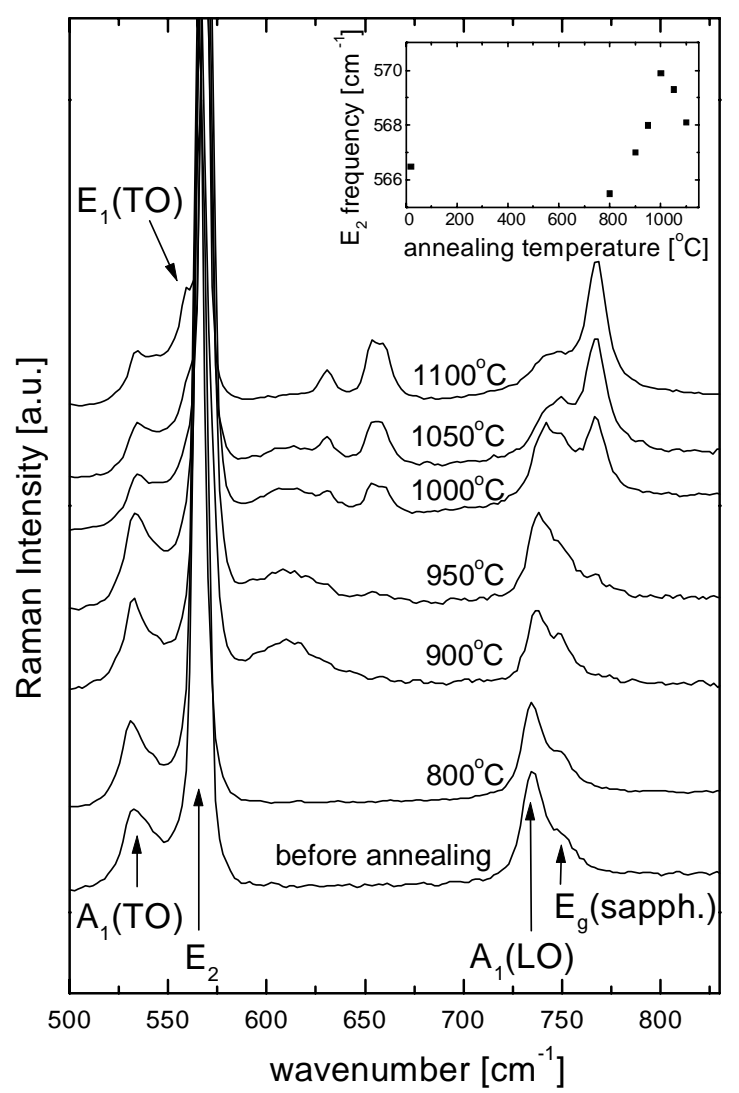

Figure 1: Raman spectra of the MBE-grown GaN sample after 20min anneals at various temperatures, recorded at room temperature in backscattering $\mathrm{Z}(\mathrm{Y}, \mathrm{Y}) \underline{\mathrm{Z}}$ geometry.

emerges at $559 \mathrm{~cm}^{-1}$ on the low-energy side of the $E_{2}$ phonon (uppermost spectrum in Figure 1). We observe a tentative decrease in the $\mathrm{E}_{2}$ phonon frequency for the $1100^{\circ} \mathrm{C}$ anneal (inset of Fig.1).

The Raman features induced by the $1000^{\circ} \mathrm{C}$ and $1100^{\circ} \mathrm{C}$ anneal are present in all polarization configurations with no evident polarization dependence. We note that the magnitude of the broad Raman peak at $610 \mathrm{~cm}^{-1}$ after annealing at $1100^{\circ} \mathrm{C}$ is sample-to-sample dependent: it apparently decreases in Figure 1, whereas for other samples a further increase was observed during the high-temperature annealing. The dramatic changes in the Raman spectrum for anneals higher than $1000^{\circ} \mathrm{C}$ go along with modifications in the surface morphology. We observe a pitting of the surface, however, the surface remains visually mirror-like. Macroscopic disorder appears.

Additional Raman experiments were performed on the MOCVD-grown GaN sample. The observed characteristic changes in the Raman spectrum during the annealing sequence are similar to the results shown in Figure 1. The temperature regimes, however, appear to be somewhat different for the MBE- and MOCVD-grown sample. Figure 2 compares Raman spectra obtained from the MOCVD- and MBE-grown GaN sample (comparable layer thickness) in the spectral region of the $\mathrm{A}_{1}(\mathrm{LO})$ phonon, after annealing the sample at a temperature of $1050^{\circ} \mathrm{C}$. The emerging distinct Raman peaks at $630 \mathrm{~cm}^{-1}, 656 \mathrm{~cm}^{-1}$ and $770 \mathrm{~cm}^{-1}$, illustrating the severe degradation of the sample, seem to be less pronounced for the MOCVD-grown GaN layer. The investigated MOCVD-grown GaN layer appears therefore to be thermally more stable than the investigated MBE-grown GaN layer. 


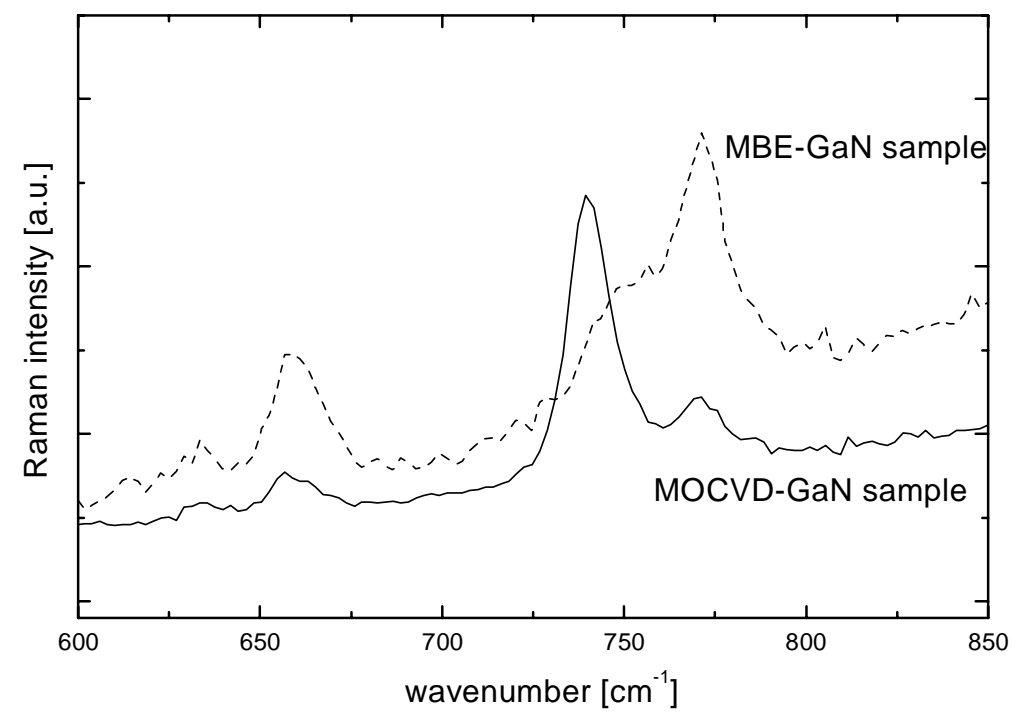

Figure 2: Raman spectra of MBE-grown GaN and MOCVD-grown GaN annealed at $1050^{\circ} \mathrm{C}$.

\section{Photoluminescence}

Figure 3 and 4 show photoluminescence spectra of the MBE-grown GaN sample recorded in the spectral region of the well-known R-lines of sapphire and the GaN bandgap, respectively, after the various high-temperature anneals. Annealing at a temperature of $1000^{\circ} \mathrm{C}$ and $1100^{\circ} \mathrm{C}$ results in a strong photoluminescence signal around $1.75 \mathrm{eV}$. Furthermore, we detect a shift and broadening of the sapphire R-lines. Figure 4 shows the photoluminescence spectrum of the MBE-grown GaN sample in the spectral region of the GaN bandgap. Before annealing we detect photoluminescence from the $\mathrm{GaN}$ bandgap at $3.4 \mathrm{eV}$. Annealing at high temperature results in a decreasing bandgap photoluminescence intensity, a broadening of the photoluminescence signal, as well as a shift of the photoluminescence maximum towards higher photon energies.

\section{DISCUSSION}

The evolution of the GaN Raman spectrum shown in Figure 1 allows us to determine and monitor noninvasively the degradation pathway of $\mathrm{GaN}$ during the annealing sequence. Three thermal stability/degradation regimes can be identified: (1) annealing temperatures up to 800$900^{\circ} \mathrm{C}$, (2) between $900^{\circ}$ and $1000^{\circ} \mathrm{C}$ and (3) higher than $1000^{\circ} \mathrm{C}$. Below $900^{\circ} \mathrm{C}$ no structural degradation of the $\mathrm{GaN}$ is evident from the Raman spectra. This is consistent with previous reports $[7,8]$. Such temperatures are well suited for high-temperature processing as well as active and passive high-temperature devices. Annealing temperatures higher than $900^{\circ} \mathrm{C}$ result in the degradation of the crystalline quality of the GaN layer, which is evident in the emerging broad Raman peak between the $\mathrm{E}_{2}$ and $\mathrm{A}_{1}(\mathrm{LO})$ phonon (Figure 1). Microscopic defects created by the high-temperature annealing result in the breakdown of the translational symmetry and the activation of the phonon density of states in the Raman spectrum, i.e., the participation of $\mathbf{q} \neq \mathbf{0}$ phonons in the Raman scattering process (disorder-induced Raman scattering). The broad Raman peak at $610 \mathrm{~cm}^{-1}$ is located in a spectral region with a high phonon density of states according to lattice dynamical calculations by Azuhata et al. [9]. It is present in all polarization configurations as expected for disorder-induced Raman scattering. Consistently, we observe a decreasing band edge photoluminescence signal during the annealing sequence as shown in Figure 4. Similar changes in the GaN photoluminescence spectrum were reported by Lin et al. [10] in 


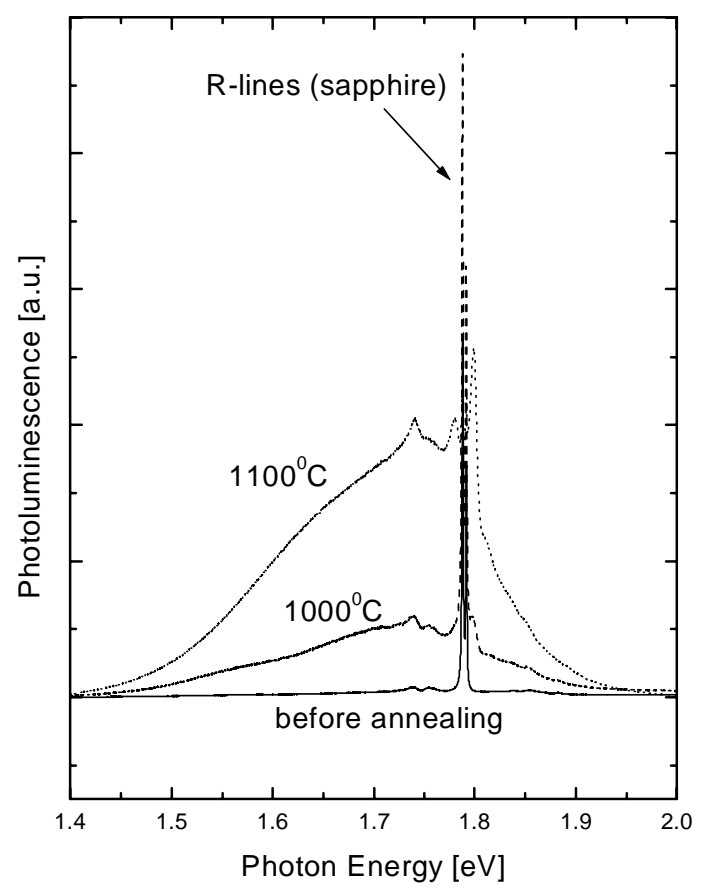

Figure 3: Photoluminescence spectrum of the MBE-grown GaN sample, after various 20min anneals.

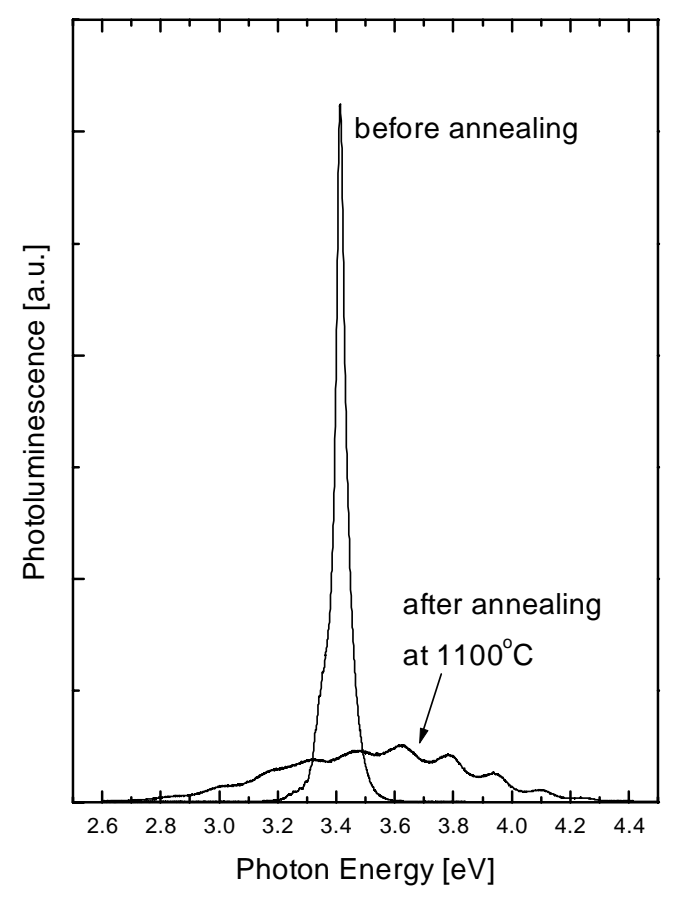

Figure 4: Photoluminescence spectrum of the MBE-grown GaN sample, after various 20min anneals.

low-temperature photoluminescence experiments on GaN samples, which were annealed at $900^{\circ} \mathrm{C}$ for $30 \mathrm{~min}$, i.e., pointing to non-radiative recombination centers. Note that no obvious modifications in the surface morphology are visible after the annealing. The observed frequency shift of the $E_{2}$ phonon by about $3 \mathrm{~cm}^{-1}$ during the annealing sequence (inset of Figure 1) reflects the appearance of internal stress [11].

Forbidden sapphire modes were observed by Barker et al. [12] at $633 \mathrm{~cm}^{-1}, 652 \mathrm{~cm}^{-1}$ and $770 \mathrm{~cm}^{-1}$ on surface-damaged sapphire. The activation of the forbidden $770 \mathrm{~cm}^{-1}$ sapphire mode for annealing temperatures higher than $1000^{\circ} \mathrm{C}$ (Figure 1) is therefore a clear indication for a degradation of the $\mathrm{GaN} / \mathrm{sapphire}$ interface during the high-temperature annealing, i.e., a reaction between $\mathrm{GaN}$ and sapphire at the interface. No GaN modes or a high GaN phonon density of states is present around $770 \mathrm{~cm}^{-1}$ and could contribute [9]. The observed photoluminescence signal at $1.75 \mathrm{eV}$ around the sapphire R-lines as well as the shift and broadening of the sapphire R-lines (Figure 4) consistently illustrate structural modifications at the GaN/sapphire interface (defects, stress) [13]. We note that no change in the Raman or the photoluminescence spectrum of sapphire was observed when a plain sapphire wafer was annealed under the same conditions. The assignment of the $630 \mathrm{~cm}^{-1}$ and $656 \mathrm{~cm}^{-1}$ Raman modes in Figure 1 is more difficult since both sapphire with its forbidden modes at $633 \mathrm{~cm}^{-1}$ and $652 \mathrm{~cm}^{-1}$ as well as GaN [9] contribute in this frequency range. Raman experiments on the side face of an annealed $6 \mu$ m-thick MBEgrown GaN layer, however, indicate that the $630 \mathrm{~cm}^{-1}$ and $656 \mathrm{~cm}^{-1}$ Raman modes mainly arise from the GaN/sapphire interface region suggesting a larger contribution from the forbidden sapphire modes. The presence of the $\mathrm{E}_{1}(\mathrm{TO})$ phonon at $559 \mathrm{~cm}^{-1}$ after the $1100^{\circ} \mathrm{C}$ anneal (Figure 1) shows an evolving misorientation of the GaN layer during the high-temperature annealing. 


\section{CONCLUSIONS}

We have demonstrated the great potential of Raman scattering to detect damage in GaN induced by high-temperature annealing. The results can be applied to monitor noninvasively damage in GaN during high-temperature processing as well as to monitor passive and active high-temperature nitride devices. Three distinct regimes of thermal damage where identified by characteristic Raman spectra. Raman scattering reveals thermal damage due to microscopic defects for anneals between $900^{\circ} \mathrm{C}$ and $1000^{\circ} \mathrm{C}$ based on the appearance of a disorder-induced Raman scattering signal between the $\mathrm{E}_{2}$ and $\mathrm{A}_{1}(\mathrm{LO})$ phonon. The evolving Raman spectrum displays the $\mathrm{GaN}$ phonon density of states. Above $1000^{\circ} \mathrm{C}$, Raman scattering probes an interfacial reaction between $\mathrm{GaN}$ and sapphire, illustrated by the activation of forbidden sapphire modes. The investigated MOCVD-grown GaN sample was found to be thermally more stable than the investigated MBE-grown GaN sample. Defect-related photoluminescence was found after the high-temperature annealing of $\mathrm{GaN}$. The $\mathrm{E}_{2}$ phonon frequency was analyzed to deduce information on internal stress. Annealing below $900^{\circ} \mathrm{C}$ has no effect on the $\mathrm{GaN}$ crystalline quality and is therefore well suitable for high-temperature applications of $\mathrm{GaN}$.

\section{ACKNOWLEDGEMENTS}

We thank G.D. Pitt (Renishaw Transducers plc.) for financial support and supplying the heating stage, H. Sands and D.N. Batchelder (University of Leeds) for support for the ultraviolet photoluminescence experiments and B. Gil for helpful discussions and encouragement.

\section{REFERENCES}

[1] S. Nakamura, M. Senoh, S. Nagahama, N. Iwasa, T. Yamada, T. Matsushita, H. Kiyoku, Y. Sugimoto, T. Kozaki, H. Umemoto, M. Sano, K. Chocho, Appl. Phys. Lett. 72, 211 (1998).

[2] Y.-K. Song, M. Kuball, A.V. Nurmikko, G.E. Bulman, K. Doverspike, S.T. Shappard, T.W. Weeks, M. Leonard, H.S. Kong, H. Dieringer, and J. Edmonds, Appl. Phys. Lett. 72, 1418 (1998).

[3] S. Nakamura, T. Mukai, M. Senoh, and N. Iwasa, Jpn. J. Appl. Phys. 31, L139 (1992).

[4] M.A. Khan, Q. Chen, R.A. Skogman, and J.N. Kuznia, Appl. Phys. Lett. 66, 2046 (1995).

[5] M. Kuball, F. Demangeot, J. Frandon, M.A. Renucci, J. Massies, N. Grandjean, R.L. Aulombard, and O. Briot, Appl. Phys. Lett. 73, 960 (1998).

[6] J.C. Zolper, M. Hagerott Crawford, A.J. Howard, J. Ramer, and S.D. Hersee, Appl. Phys. Lett. 68, 200 (1996).

[7] J. Hong, J.W. Lee, J.D. MacKenzie, S.M. Donovan, C.R. Abernathy, S.J. Pearton, and J.C. Zolper, Semicond. Sci. Technol. 12, 1310 (1997).

[8] H. Siegle, G. Kaczmarczyk, L. Filippidis, A.P. Litvinchuk, A. Hoffmann, and C. Thomsen, Phys. Rev. B 55, 7000 (1997).

[9] T. Azuhata, T. Matsunaga, K. Shimada, K. Yoshida, T. Sota, K. Suzuki, and S. Nakamura, Physica B 219\&220, 493 (1996).

[10] M.E. Lin, B.N. Sverdiov, and H. Morkoç, Appl. Phys. Lett. 63, 3625 (1993).

[11] F. Demangeot, J. Frandon, M.A. Renucci, O. Briot, B. Gil, R.-L. Aulombard, MRS Internet J. Nitride Semicond. Res. 1, 23 (1996).

[12] A. S. Barker, Jr., Phys. Rev. 132, 1474 (1963).

[13] L. Grabner, J. Appl. Phys. 49, 580 (1978). 